\title{
Identification and Evaluation of an Appropriate Housekeeping Gene for Real-Time Gene Profiling of Hepatocellular Carcinoma Cells Cultured in Three- Dimensional Scaffold
}

\author{
Anjana Kaveri Badekila \\ Nitte University \\ Praveen Rai \\ Nitte University \\ Sudarshan Kini ( $\nabla$ sudarshan.kini@nitte.edu.in ) \\ Nitte University https://orcid.org/0000-0001-5253-9938
}

\section{Research Article}

Keywords: 3D culture, hepatocellular carcinoma cell line, housekeeping gene, quantitative real time polymerase chain reaction

Posted Date: July 8th, 2021

DOI: https://doi.org/10.21203/rs.3.rs-490561/v2

License: (c) (i) This work is licensed under a Creative Commons Attribution 4.0 International License.

Read Full License

Version of Record: A version of this preprint was published at Molecular Biology Reports on October 19th, 2021. See the published version at https://doi.org/10.1007/s11033-021-06830-y. 


\section{Abstract}

Assessing an optimal reference gene as an internal control for target gene normalization is important during quantitative real time polymerase chain reaction (RT-qPCR) of three-dimensional cell culture. Especially, gene profiling of cancer cells under a complex 3D microenvironment in a polymer scaffold provides a deeper understanding of recapitulation of in vivo tumors. In this aspect, expression of six housekeeping genes (HKG's): glyceraldehyde-3-phosphodehydrogenase (GAPDH), $\beta$-actin (ACTB), beta-2microglobulin (B2M), 18S ribosomal RNA (18S rRNA), peptidyl-propyl-isomerase A (PPIA), and ribosomal protein L13 (RPL-13)) during the monolayer culture (two-dimensional), and alginatecarboxymethylcellulose scaffold based three-dimensional (3D) cell culture conditioned up to 21 days was analysed for hepatocellular carcinoma (Huh-7) cell line. The real-time gene expression using RT-qPCR of HCC spheroids in 3D culture were analyzed by determining the primer efficiency, melting curve and quantification cycle analysis of the selected candidate HKG's. Further, RT-qPCR data were validated using analysis softwares i.e., geNorm and NormFinder for statistical significance. The study indicated RPL-13, $18 \mathrm{~S}$ rRNA and B2M to be stable among selected referral HKG candidates and considered them as potential internal controls during varying cell culture conditions.

\section{Introduction}

Emerging studies indicate hepatocellular carcinoma (HCC), a primary liver cancer, as one of the leading causes of cancer in the world. Application of hepatocellular carcinoma cells for disease modeling in vitro is significantly vital in cancer research due to the dysfunctional liver capacity of HCC in vivo, and to reduce an extensive application of the animal model. A common function of a major internal organ like the liver in vivo is to execute the metabolism of macro/micronutrients, synthesise serum proteins like albumin, and eliminate foreign compounds by transforming them into water-soluble excretions. In this regard, dysfunction of the liver that executes over 500 functions by HCC is a major hurdle for other interrelated functions; hence, in vitro liver disease models are majorly being applied for inventing novel therapeutic avenues.

Presently, hepatocellular carcinoma cells like HepG2, Huh-7 cells etc., are majorly being applied in propagating hepatitis $\mathrm{B}$ virus for anti-viral research, disease modelling, discovering novel therapeutic approaches, and drug toxicity testing using two-dimensional cell culture [1, 2]. Also, most of the cell culture techniques today are performed in two dimensions due to the ease, convenience and higher cellular viability associated with these systems. However, the adaption of cells in two-dimensions (2D) forces them to alter their gene functionality, thereby deviating results from the expected behaviour in vivo. Currently many research works are being focused on developing scaffold-based three-dimensional (3D) liver cancer models in vitro using natural polymers like alginate, carboxymethylcellulose, chitosan, etc., with an aim towards creating a precise disease model [3]. The application of 3D culture allows integration of heterogeneous cells clustered together to form a liver tumor that recapitulates the native characteristics of hepatocellular carcinoma cells. A scaffold-based 3D cell culture allows cells in vitro to grow and proliferate in a manner alike in vivo environment [4]. 
Analysing the gene expression of cells/spheroids grown in three-dimensional cell cultures, and at different time phases is significantly important for disease modeling. This is because, cellular characteristics like migration, proliferation, differentiation, and metabolism varies over their life span. For elucidating cellular characteristics, selection of a suitable housekeeping gene for normalization of target gene expression is important in gene expression studies. One of the popular techniques applied for gene expression study is quantitative real time polymerase chain reaction (RT-qPCR) due to its simplicity, specificity, and sensitivity [5]. Herein, the amplification of a target gene is monitored by quantitatively measuring the number of copies of a gene present in a sample by intercalating the amplified DNA with a fluorescent TaqMan probe/dye. Generally, the gene expression of a target is investigated with relation to a reference or endogenous control called as housekeeping gene for accurate normalization of expression levels. The normalization of the target gene is important to correct the quantity and quality differences among the culture samples by using a native endogenous control called as a housekeeping gene (HKG) $[6,7]$.

A housekeeping gene is a reference calibrator whose threshold cycle $\left(C_{t}\right)$ value may ideally remain constant throughout the treatment or life span of a biological system. In eukaryotic models, application of either of the housekeeping genes like ribosomal RNA based 18S rRNA, ribosomal protein L13 (RPL-13), or a gene contributing towards cytoskeleton organisation like $\beta$-actin (ACTB) are popular. Other common HKGs include cellular metabolism enzymes like glyceraldehyde-3-phosphodehydrogenase (GAPDH) responsible for glucose breakdown, peptidyl-propyl-isomerase A (PPIA) for intracellular signalling, and an immune complex molecule like $\beta$-2-microglobulin (B2M) regulating tumor growth $[7,8]$. Over the years, it has been proven that commonly used HKG's like GAPDH and $\beta$-actin tends to show alteration in gene expression and are considered unreliable in target gene normalization due to large variation in their mean threshold cycle values at different experimental conditions such as hypoxia induction [9], simulated differentiation/regeneration [10], tissue specific alteration, generational gap, [6] and untoward micro-RNA interference [11]. Therefore, it is indispensable to identify a reference calibrator that provides powerful insight on the relative difference in the gene expression of target genes in 3D cell cultures [12]. As per our knowledge, research in 3D cell culture is still in its nascent stage and many studies haven't focused on identifying an ideal HKG for genotypic studies of the hepatocellular carcinoma cell line. Also, owing to the complexities associated with three-dimensional cell culture, it is pertinent to investigate the housekeeping genes for accurate quantification of target gene expression. In this aspect, a study compared different HKG's to normalize real time PCR (qPCR) data in senescence studies of Mesenchymal stem cells (MSC) and interpreted GAPDH and $\beta$-actin to be least stable compared to PPIA, RPL-13 and B2M [10]. Similarly, another study showed the variability of HKG like GAPDH, ACTB, 18S rRNA, B2M, TATA-binding box protein etc. in 3D scaffold cultured chondrocytes during hypoxia, to conclude a change in gene expression for HKGs in variable culture conditions by melting curves analysis, and stability assessment software applications (geNorm and NormFinder) [13]. However, in 3D culture systems, investigating the cellular growth kinetics, proliferation, and metabolism during different time intervals is important for maintenance and enrichment of spheroids. In one such instance, a study developed alginate scaffolds for human glioblastoma cell line cultured at different time intervals $(7,14$, and 21 days). The influence of 
alginate scaffolds on cellular viability, differentiation and proliferation during different time points was profound up to 21 days [14]. Hence, longevity of 3D culture period necessitates a need for analysing appropriate HKG during different time intervals. In this study, we aimed to identify appropriate internal control in quantitative real time polymerase chain reaction using popular HKG's like GAPDH, ACTB, 18S rRNA, PPIA, B2M, and RPL-13.

\section{Materials And Methods}

Hepatocellular carcinoma cell line (Huh-7) (Cell repository, National Centre for Cell Science, Pune, India), DMEM complete growth media (D5796, Sigma Aldrich, USA), fetal bovine serum (F2442, Sigma Aldrich, USA), trypsin-EDTA (T4049, Sigma Aldrich, USA), antibiotic-antimycotic (P4333, Sigma Aldrich, USA), gentamycin sulphate (G13970, Sigma Aldrich, USA), phosphate buffered saline (P3813, Sigma Aldrich, USA), acridine orange (A6014, Sigma Aldrich, USA), TRIzol reagent (162710, Invitrogen, USA), chloroform (AS039, Himedia, India), analytical grade ethanol (Hayman, UK), isopropanol (00270, Lobachemie, India), and quantitative real-time PCR kit (RR086A, TaKaRa, Japan) were the materials and reagents used in this study.

\section{Cell culture}

Huh-7 cells were grown in DMEM complete growth media supplemented with $10 \%$ fetal bovine serum, LGlutamine, antibiotic-antimycotic solution and gentamycin sulphate. Cells were grown under optimum culture conditions as described previously [15]. Cells were dissociated by using $0.25 \%$ Trypsin-EDTA solution and cell pellet was collected by centrifugation before seeding in 2D and 3D cultures. The Huh-7 cells were allowed to grow in a $5 \% \mathrm{CO}_{2}$ incubator maintained at $37^{\circ} \mathrm{C}$.

\section{D cell culture}

Three-dimensional culture of Huh-7 were prepared using alginate-CMC cryogel based scaffold. Briefly, the cells were re-suspended in DMEM complete growth media with a final cell count of $1 \times 10^{5} \mathrm{cells} / \mathrm{ml}$. Meanwhile, the polymer solution was prepared by dissolving alginate and $C M C$ in 1:1 ratio with double distilled water. The prepared solution was sterilized and stored at $4^{\circ} \mathrm{C}$. Likewise, $2 \%$ calcium chloride was prepared in autoclaved water and syringe filtered $(0.2 \mu \mathrm{m}$ pore size) to pass through a nozzle. Using Nunc's $35 \times 10 \mathrm{~mm}$ cell culture dish, AL-CMC bioink solution was added and the lid was closed and tightly sealed with a parafilm (Bemis, USA). After freezing the polymer solution at $-80^{\circ} \mathrm{C}$ for $24 \mathrm{~h}$, instant thawing with calcium chloride was performed under sterile conditions. The fabricated scaffold was washed thrice with 1X PBS and DMEM complete growth media was added prior to incubation at $37^{\circ} \mathrm{C}$ for enhancing growth conditions of the spheroid [3].

\section{Nucleic acid staining}

The utilized DMEM media was aspirated from the Huh-7 cells grown in 2D culture and spheroid containing scaffold within the culture dish. The scaffold matrix was dissolved using algimatrix dissolving 
solution (A1134001-Gibco, USA). Live staining of Huh-7 cells grown in 2D and alginate-CMC based 3D cell culture was performed using acridine orange dye (Sigma A6014, USA). The utilized DMEM media was aspirated from the scaffold within the culture dish and stained with $500 \mu \mathrm{l}$ of $30 \mu \mathrm{M}$ acridine orange stain for 15-20 min. The spheroids were washed thrice with 1X PBS gently and observed under a fluorescent microscope (BX53-Olympus, Japan) [16, 17].

\section{RNA extraction}

Total RNA was extracted from Huh-7 cells grown in a polystyrene flask (2D culture) and alginate-CMC (3D culture) at different time intervals (7, 14, and 21 days). In 3D culture, dissociation of the matrix was performed using algimatrix dissolving solution prior to RNA extraction. RNA extraction was performed using TRIzol reagent (Invitrogen, USA) following the manufacturer's instructions. The quality and quantity of RNA was estimated using nanophotometer (N60-Implen, USA) [18, 19].

\section{Designing of primers}

The primers for HKG's were designed using real time PCR (TaqMan) primer designing software, GenScript (Piscataway, NJ) (https://www.genscript.com/tools/real-time-pcr-taqman-primer-design-tool). The sequences for all the gene sequences were retrieved from GenBank database (www.ncbi.nlm.nih.gov). The exon boundaries in eukaryotic gene code were defined and submitted in raw format. The software provided an advantage to pick primer/probe crossing exon junction. The criteria for choosing an appropriate primer were based on a length of 18-21 bases and an amplicon size of up to 200 base pairs. Further, the obtained primer design was matched with a model organism using BLAST analysis. The gene description, primer sequences and amplicon sizes used in this study are represented in Table 1. 
Table 1

Represents the primer sequences of different housekeeping genes

\begin{tabular}{|c|c|c|c|c|c|}
\hline $\begin{array}{l}\text { Gene } \\
\text { name }\end{array}$ & Function & $\begin{array}{l}\text { Accession } \\
\text { number }\end{array}$ & $\begin{array}{l}\text { Primer } \\
\text { code }\end{array}$ & Primer sequences $\left(5^{\prime}-3^{\prime}\right)$ & $\begin{array}{l}\text { Amplicon } \\
\text { size (bp) }\end{array}$ \\
\hline \multirow[t]{2}{*}{$\begin{array}{l}\text { 18S } \\
\text { rRNA }\end{array}$} & \multirow{2}{*}{$\begin{array}{l}\text { Synthesis of } \\
\text { ribosomal } \\
\text { proteins }\end{array}$} & \multirow[t]{2}{*}{ NC_000021.9 } & $\begin{array}{l}\text { 18S } \\
\text { rRNAF }\end{array}$ & AGAAACGGCTACCACATCCA & \multirow[t]{2}{*}{121} \\
\hline & & & $\begin{array}{l}18 \mathrm{~S} \\
\text { rRNAR }\end{array}$ & TACAGGGCCTCGAAAGAGTC & \\
\hline \multirow[t]{2}{*}{ PPIA } & \multirow{2}{*}{$\begin{array}{l}\text { Intracellular } \\
\text { signalling }\end{array}$} & \multirow[t]{2}{*}{ NC_000007.14 } & PPIAF & TTTTCATCTGCACTGCCAAG & \multirow[t]{2}{*}{160} \\
\hline & & & PPIAR & TCGAGTTGTCCACAGTCAGC & \\
\hline \multirow[t]{2}{*}{ RPL-13 } & \multirow{2}{*}{$\begin{array}{l}\text { Synthesis of } \\
\text { ribosomal } \\
\text { proteins }\end{array}$} & \multirow[t]{2}{*}{ NC_000016.10 } & RPL-13F & GGACCATCGGCATTTCTGTG & \multirow[t]{2}{*}{97} \\
\hline & & & RPL-13R & AGTTTGGAGCGGTACTCCTT & \\
\hline \multirow[t]{2}{*}{ GAPDH } & \multirow{2}{*}{$\begin{array}{l}\text { Cellular } \\
\text { metabolism } \\
\text { enzyme } \\
\text { responsible } \\
\text { for glucose } \\
\text { breakdown }\end{array}$} & \multirow[t]{2}{*}{ NC_000012.12 } & GAPDHF & GGACTCATGACCACAGTCCA & \multirow[t]{2}{*}{157} \\
\hline & & & GAPDHR & TCAGCTCAGGGATGACCTTG & \\
\hline \multirow[t]{2}{*}{ ACTB } & \multirow{2}{*}{$\begin{array}{l}\text { Contributes } \\
\text { towards } \\
\text { cytoskeleton } \\
\text { organisation }\end{array}$} & \multirow[t]{2}{*}{ NC_000007.14 } & ACTBF & CCCTGGAGAAGAGCTACGAG & \multirow[t]{2}{*}{106} \\
\hline & & & ACTBR & TCCATGCCCAGGAAGGAAG & \\
\hline \multirow[t]{2}{*}{ B2M } & \multirow{2}{*}{$\begin{array}{l}\text { Immune } \\
\text { complex } \\
\text { molecule }\end{array}$} & \multirow[t]{2}{*}{ NC_000015.10 } & B2MF & GCGCTACTCTCTCTTTCTGG & \multirow[t]{2}{*}{133} \\
\hline & & & B2MR & TGTCGGATGGATGAAACCCA & \\
\hline
\end{tabular}

\section{Quantification of the housekeeping gene}

Real time PCR was performed to quantify the level of housekeeping gene expression in hepatocellular carcinoma cell line. One-step RT-qPCR assay was performed with the final volume of $25 \mu$ using PrimeScript one-step RT-qPCR kit (TaKaRa Biotech, Japan) as per the manufacturer's instructions. The reaction mixture contained $12.5 \mu \mathrm{l}$ of $2 x$ SYBR green master mixes (RR0086A, TaKaRa, Japan), $300 \mathrm{nM}$ forward and reverse primers, and $1 \mu \mathrm{l}$ of total RNA $(50 \mathrm{ng} / \mu \mathrm{l})$. The reverse transcription was conditioned at a temperature of $42^{\circ} \mathrm{C}$ for 5 minutes, and $95^{\circ} \mathrm{C}$ for 10 seconds during one cycle followed by a PCR reaction at $95^{\circ} \mathrm{C}$ for $5 \mathrm{~s}$, while the annealing temperature was ranging from $55-64^{\circ} \mathrm{C}$ for 20 seconds (40 cycles). A melting curve analysis was performed by heating at $95^{\circ} \mathrm{C}$ for 15 seconds, $60^{\circ} \mathrm{C}$ for 1 minute and finally $95^{\circ} \mathrm{C}$ for 15 seconds to verify the PCR product. The reaction was performed using CFX96- real time system (BioRad, USA). Based on the quantification/threshold cycle value $\left(\mathrm{C}_{t}\right)$ obtained, the expression stability of HKG's was assessed.

The primer efficiency applied for the target amplification was evaluated in serial dilutions $(1 / 10,1 / 100$, $1 / 1000,1 / 10000$, and 100000) using triplicates. Following quantitative real time PCR (RT-qPCR), the 
threshold cycle $\left(C_{t}\right)$ values obtained at different RNA concentrations $(0.01-100 \mathrm{ng} / \mu \mathrm{l})$ was linearly plotted to determine the slope and correlation coefficient $\left(R^{2}\right)$ [10]. After determining the corresponding values, primer efficiencies were calculated by the following equation, Eq. (1).

$$
E=\left[10^{\wedge(-1 / \text { slope })}-1\right] \times 100
$$

\section{Analysis of gene expression}

The stability of all the HKG's obtained after RT-qPCR were analysed using two softwares: NormFinder software, version 0.9 (Trial version) and geNorm algorithm integrated in qbaseplus software, version 2.4 (Trial version). Both software integrated the raw threshold cycle values obtained specifically using CFX96BIO-RAD real time system (BioRad, USA) in Microsoft excel format. The NormFinder software calculated the stability index by applying a statistical approach of analysis of variance (ANOVA) between the expression values of all housekeeping genes that were logarithmically transformed $\left(\log C_{t}\right)$. The proportion of intra and inter group variation on overall variation was taken into consideration while deriving a normalization factor (NF). In situations where no optimal single reference gene was found, application of multiple reference genes comes into the scenario with a rationale: variation in the averaging of multiple genes was smaller than a variation among inter and intra subgroups of individual housekeeping genes. In this study, the geometric mean value of sample triplicate was applied for stability measure of candidate HKG during 2D, and 3D (7, 14, and 21 days) culture, as different time points were considered as grouping variables [20]. The statistical framework ranked candidate housekeeping genes enabling the evaluation of systemic error introduced during the period of gene normalization [21]. For further evaluation of housekeeping genes, specifically during different time intervals of 3D culture, expression analysis software geNorm was applied, whose algorithm was based on the criterion that two ideal housekeeping genes have a minimal expression ratio across the investigated sample set regardless of cell type or treatment condition. The algorithm of the geNorm software was conditioned to calculate the standard deviation of the logarithmically transformed expression ratio across 3D culture samples for a particular gene relative to other genes under investigation. General specifications for the application of geNorm software required a minimum of three reference genes and two sample types. The software statistically applied a program to analyse the average stability measure (M) of each housekeeping gene with respect to the expression of other housekeeping genes and ranked them based on stepwise exclusion method. Further, geNorm software eliminated the genes with the highest $M$ value from the panel and recalculated new $\mathrm{M}$ values for the remaining genes until reaching the last two genes with the smallest $M$ value. The lower the $M$ value, the higher stability was considered for the candidate gene [13, 22]. Hence, the most stable housekeeping gene was considered to be a gene with the lowest $M$ value and the mathematical model, thus, evaluated the stability of the housekeeping genes by a robust measure for further confirmation. 


\section{Results}

\section{Nucleic acid staining}

Live imaging of Huh-7 cells seeded scaffolds confirmed the formation of tumour spheroids in vitro due to differential staining of DNA and RNA. This is because acridine orange selectively binds nucleic acids by intercalation of DNA or RNA. Also, the association of acridine orange with RNA showed red fluorescence $\left(\boldsymbol{\lambda}_{\text {excitation }}=460 \mathrm{~nm}\right.$ blue and $\left.\boldsymbol{\lambda}_{\text {emission }}=650 \mathrm{~nm}\right)$, while with DNA or double-stranded RNA, acridine orange

showed green fluorescence $\left(\boldsymbol{\lambda}_{\text {excitation }}=502 \mathrm{~nm}\right.$ blue and $\left.\boldsymbol{\lambda}_{\text {emission }}=525 \mathrm{~nm}\right)$ [23]. The acridine orange stained hepatocellular carcinoma cells cultivated in 2D (Fig. 1(a)) showed monolayer formation, and 3D culture during different time intervals of 7, 14 and 21 days showed spheroid formation (Fig. 1(b-d)). Also, a confirmation on the influence of alginate-carboxymethylcellulose scaffold on cellular viability of hepatocellular spheroids with respect to time was observed [3] i.e., a decrease in spheroid number linking the degradation of scaffold matrix. However, the longevity of spheroids up to 21 days necessitated gene expression studies based on time, which is required for the enrichment and maintenance of 3D culture.

\section{Gene profiling of housekeeping genes}

The melting curve analysis was performed on candidate HKG's like GAPDH, ACTB, PPIA, RPL-13, B2M and $18 \mathrm{~S}$ rRNA. The analysis showed an emergence of single fluorescence peak (indicative of a single PCR product) for all the primers applied in this study (Fig. 2(a-f)). The genetic content of hepatocellular carcinoma cells grown in 2D and 3D cultured samples showed an absolute fluorescence peak at a melting temperature of $81^{\circ} \mathrm{C}$ for B2M (Fig. 2 (a)), $88^{\circ} \mathrm{C}$ for RPL-13 gene (Fig. 2(b)), $86^{\circ} \mathrm{C}$ for $18 \mathrm{~S}$ rRNA (Fig. 2(c)), $83^{\circ} \mathrm{C}$ for ACTB (Fig. 2(d)), $87^{\circ} \mathrm{C}$ for GAPDH (Fig. 2(e)), and $84^{\circ} \mathrm{C}$ for PPIA gene (Fig. 2(f)), and, respectively.

Further, amplification efficiencies of the primers were examined by deriving the slope of threshold cycle values plotted against log-transformed values obtained for different RNA dilutions. The linear plot and slope of the obtained data indicated an efficient amplification of the housekeeping genes affects RTqPCR analysis. The efficiency of the primers obtained through the analysis was summarized with $E$ value of approximately $94-108 \%$ (Table 2 ) and correlation coefficient $R^{2}>0.98$ for 18 S rRNA, B2M, RPL-13, ACTB, PPIA and GAPDH (Fig. 3(a)). 
Table 2

Summarizes the efficiencies of all primers applied in this study

\begin{tabular}{|llll|}
\hline Gene name & Slope & $\mathbf{R}^{2}$ & Efficiency \\
\hline 18S rRNA & -3.42 & 0.99 & 94.1 \\
\hline RPL-13 & -3.3 & 0.99 & 100.9 \\
B2M & -3.2 & 0.99 & 105.3 \\
GAPDH & -3.1 & 0.98 & 108.9 \\
ACTB & -3.2 & 0.99 & 99.5 \\
PPIA & -3.1 & 0.98 & 108.9 \\
\hline
\end{tabular}

For stability analysis, a comparison of threshold cycle values for the candidate HKG's was performed. The mRNA level and variable expression of the HKG candidates were analysed using their mean threshold cycle/ quantification cycle value $\left(\mathrm{C}_{t}\right)$. The criterion for choosing a stable housekeeping gene was based on achieving an approximate or near-constant quantification cycle value during monolayer $2 \mathrm{D}$ culture and at different time intervals of 3D culture. While performing the RT-qPCR experiment, nearconstant $C_{t}$ value for four different samples of candidate HKG across different culture conditions indicated stable and reliable housekeeping gene. The mean threshold cycle $\left(C_{t}\right)$ values $(95 \%$ of class interval: lower - upper value) at different time intervals of 7, 14, and 21 days of culture was represented in Fig. 3(b) (Data in supplementary file, Table 1S) for different HKG. By comparing the HKG candidates like 18S rRNA, PPIA, RPL-13, GAPDH, B2M, and $\beta$-actin, we found 18S rRNA, B2M, and RPL-13 genes to show a stable threshold cycle value. While other commonly applied housekeeping genes for eukaryotic cells like GAPDH (25 (95\% Cl: 22.8-27.2)), $\beta$-actin (29 (95\% Cl: 26.2-31.8)), and PPIA (27(95\% Cl: 25.2-28.8)) deemed to show variations in threshold cycle $\left(C_{t}\right)$ values. This variation is mainly attributed to substantial changes of culture condition for 3D spheroids maintained in vitro (13). Hence, our finding suggests that the application of RPL-13 with a mean $\mathrm{C}_{\mathrm{t}}$ value of $25(95 \% \mathrm{Cl}$ : $24.2-25.8)$, $18 \mathrm{~S}$ rRNA (14 (95\% Cl: $12.8-$ $15.2)$ ), and B2M (21 (95\% Cl: 19.9-22.1)) during both types of cultures at different time intervals $(7,14$, and 21 days) (Fig. 3(b)) is an excellent choice, regardless of the culture condition/treatment. Therefore, RPL-13, 18S rRNA and B2M with stable $C_{t}$ values are applicable as normalization genes for gene expression studies of HCC cell line in 3D culture and is defined by the specificity in melting curve analysis (Fig. 2), efficient amplification (Fig. 3(a)), and near constant threshold cycle values ( $\left.C_{t}\right)$ (Fig. 3 (b)).

\section{Stability of HKG using NormFinder and geNorm analysis}

The NormFinder analysis was conducted on four samples (2D culture and 3D culture (at 7, 14 and 21 days)), while geNorm analysis was conducted for three samples based on different time intervals of 3D culture (at 7, 14 and 21 days) for six reference genes. The analysis software represented the ranking of HKG's based on their stability. The NormFinder software converted the median log transformed values of 
threshold cycle values using its inbuilt algorithm, while geNorm software determined the stability of HKG based on their average expression stability value (M) obtained from a summation of geometric means. A lower $M$ value indicated a stable gene expression for the representative HKG's [23]. The stability of the housekeeping gene determined by geNorm and NormFinder software were represented in Fig. 4. With NormFinder analysis, RPL-13 with a stability index value of 0.033 , and B2M with 0.035 were most stable compared to other genes like 18S rRNA, GAPDH, ACT-B, and PPIA showing a higher range of values of up to 0.15 (Fig. 4(a)). In this analysis, we combined the expression of both 2D and 3D culture and ranked the six housekeeping genes as follows: RPL-13 > B2M > 18S rRNA > GAPDH > ACTB > PPIA (Data in supplementary file, Table 2S). Hence, NormFinder considered RPL-13 to be the most stable housekeeping gene for different culture conditions of 2D and 3D culture. Further, geNorm HKG stability analysis software ranked the selected candidates as follows for 3D cell culture: B2M > 18S rRNA > RPL-13 > PPIA > GAPDH > ACTB (Data in supplementary file, Table 3S). According to geNorm analysis, the reference target stability $M \leq 1$ was considered optimal for heterogeneous samples like treated cultured cells, biopsies of cancer, or samples of different tissues. However, the variability among different sequential normalization factors among different HKG was high (Fig. 4 (b)). Hence, the application of two or more reference genes with the lowest $M$ value was considered useful in accurate normalization compared to the use of a single reference gene.

\section{Discussion}

Presently, 3D cell culture represents a suitable in vitro liver cancer model due to the possibility of replicating natural mechanisms of the hepatocytes as observed in vivo. The biomimicking capacity of 3D cell culture provides a superior alternative from 2D culture in exploring cancer mechanisms, hypoxia, epithelial to mesenchymal transition of cells, and pharmacokinetics of novel therapeutic agents without ethical concerns associated with animal models. To establish a 3D culture, the application of a natural polymer like alginate-CMC based scaffold is popular due to the lack of complexity in synthesis, low immunogenic or toxic response to cells, and biocompatibility [17, 24]. Moreover, alginate blended carboxymethylcellulose scaffold preparation provides better water holding capacity and a non-adherent surface that allows the cells to be aggregated and form 3D spheroids. Some studies also suggest the lack of native signalling factors in alginate-based scaffolds for cells reduces ligand-receptor interaction and thus provides an advantage of non-surface-binding during spheroid formation [25]. Therefore, the use of alginate-CMC scaffolds supports the growth of hepatocellular carcinoma spheroids and longevity up to 21 days is evident from acridine orange staining in Fig. 1. The study showed a variation of culture condition and time alters spheroid characteristic associated with its enrichment and maintenance.

Investigating the gene functionality is of great relevance in characterisation of 3D spheroids using RTqPCR. However, prior to performing RT-qPCR based genotypic studies for target genes, validation of the appropriate HKG is needed for accurate normalization, which imparts precise relative quantification results. To the best of our knowledge, this is the first study to determine optimal internal control for HCC cell line cultured at different time intervals in 3D scaffold of alginate-carboxymethylcellulose polymerbased origin. A suitable selection of candidate housekeeping genes like GAPDH, ACTB, 18S rRNA, PPIA, 
RPL-13, and B2M from previous literature entailed a broader investigation with primer efficiency analysis, melting curve analysis, geNorm, and NormFinder software-based stability analysis. During the melting curve analysis, the emergence of a single fluorescence peak at a particular melting temperature for 2D culture and different time intervals of 3D culture, as evident in Fig. 2 was indicative of the presence of a pure and single type of amplicon as an end product. Further, primers having an optimal efficiency are critical indicators of RT-qPCR performance, as relative gene expression of target gene normalized against a reference calibrator is calculated with $2^{-\Delta \Delta C t}$ method. An acceptable range for amplification efficiency of $90-110 \%$ with a $R^{2}$ value $>0.98$ during linear regression coefficient derivation shown in Fig. 3 (a). Some studies previously conducted imply that until and unless an approximate $100 \%$ efficiency is achieved, application of $2^{-\Delta \Delta C t}$ method is not the most accurate description of fold change [26]. Hence, primer designing, and amplification efficiency are crucial for reliable quantitative real time PCR. However, for selecting an optimal candidate reference gene, further evaluation of threshold cycle $C_{t}$ value was necessary. For four different sample types (2D, 3D (7 days), 3D (14 days), and 3D (21 days)), the amplitude in gene expression ranges more than 3-4 cycles pointed out great variability in heterogeneous cancer samples shown in Fig. 3(b). Previously, a study indicated heterogeneous cancer tissues showed approximately 4 cycles of larger expression in all the housekeeping genes during raw $C_{t}$ value investigation for determining the stability of a malignant tumour-like glioblastoma with non-neoplastic cells [27]. In this study, RPL-13, 18S rRNA and B2M showed stable $C_{t}$ values across different culture conditions.

Earlier, without much validation, GAPDH and ACTB were the most applied housekeeping genes for target gene normalization. Many studies, however, decline the prior assumption of their expression stability remaining constant throughout a variable culture condition $[10,11]$. Especially in the case of hepatocytes, breakdown of glucose and the role of the glycolytic enzyme in energy metabolism down-regulates GAPDH expression level during 3D culture [28]. Similarly, another HKG like ACTB is an integral component of cellular structure and a rearrangement in 3D spheroid alters its gene expression [29]. However, expression of $18 \mathrm{~S}$ rRNA, PPIA, RPL-13, and B2M are stably expressed among altered culture conditions of animal cell culture and have been validated in previous studies as well [8, 30,31]. For further analysis of the housekeeping genes, a robust measure using NormFinder showed the previously assumed HKGs to be stable among different culture conditions. However, even though geNorm analysis indicated RPL-13, 18S rRNA, and B2M to be moderately stable HKGs for 3D culture, their ranking nature differed from NormFinder analysis due to variation in sequential normalization factors and the results were thus considered trivial. Among the two approaches, the NormFinder follows a descriptive statistical approach for housekeeping gene selection compared to geNorm. Also, NormFinder considers both inter and intra group variation to evaluate stability while geNorm determines pairwise standard deviation of $C_{t}$ values of all genes excluding the HKG with the lowest stability, followed by another repetition of the total process until two genes remain to be most stable $[32,33]$. Nonetheless, the suitability of the statistical method differs based on the experimental setting. However, there is no consensus on the statistical approach to select the best housekeeping genes nor on the application of the housekeeping genes for RT-qPCR data normalization. This study verified RPL-13, B2M, and 18S rRNA to be stably expressed genes among all 
the six candidates housekeeping gene. Since the PCR evolution, the application of a single housekeeping gene that is most stable for normalization is conventionally acceptable. However, the implication of averaging the $\mathrm{C}_{t}$ values of two or more housekeeping genes for the normalization of target genes provides superior accuracy.

\section{Conclusion}

In 2D and 3D cell culture, the stability of candidate housekeeping gene should be periodically evaluated for cancer cells before real time PCR experiments with a gene of interest. Still, previous studies on target gene expression in other cancer cell lines like ovarian or prostate under hypoxic condition estimated reference genes like $18 \mathrm{~S}$ rRNA, and B2M to be most stable. In the current study, validation of an appropriate housekeeping gene was performed for liver cancer cell line cultured in different time intervals of 3D culture. According to this study, alginate-carboxymethylcellulose (CMC) polymer-based scaffold is biocompatible for the spheroid formation and their longevity is observed up to 21 days. For elucidating the natural cellular mechanisms, analysing the gene expression is one of the main criteria. In this aspect, choosing the best housekeeping gene for target gene normalization is considerably advantageous in obtaining accurate results. RPL-13, 18S rRNA, and B2M are validated as stable and reliable housekeeping gene for normalizing the expression of a target gene in cell culture experiments with Huh-7 cell line maintained under variable culture conditions. During variable culture conditions like hypoxia-normoxia, time-dependent changes, and drug dose-dependent studies in 3D culture, we recommend RPL-13, 18S rRNA and B2M as the best housekeeping genes for real time PCR analysis. However, the stability of the housekeeping genes applied in this study may vary for other cell types or culture conditions. Hence, a thorough exploration of a reliable housekeeping gene prior to performing real time PCR is necessary for accurate normalization of gene expression.

\section{Abbreviations}

HKG

Housekeeping gene

2D culture

Two-dimensional culture

3D culture

Three-dimensional culture

$\mathrm{HCC}$

Hepatocellular carcinoma

$18 S$ rRNA

$18 \mathrm{~S}$ ribosomal RNA

RPL13

Ribosomal protein L13

ACTB 
$\beta$-actin

GAPDH

Glyceraldehyde-3-phosphodehydrogenase

PPIA

Peptidyl-propyl-isomerase A

B2M

B-2-microglobulin

\section{Declarations}

\section{Conflict of interest}

No conflicts to declare.

\section{Author contribution statement}

SK: Conceptualization, writing and editing of the manuscript, AKB: Writing and original draft preparation, PR: Editing and proofreading of the manuscript.

\section{Acknowledgement}

We profusely thank Nitte (Deemed to be University) for the infrastructure and laboratory facility. A.K.B is thankful to Nitte (Deemed to be University) for the PhD fellowship. Authors are also thankful to the Director, NUCSER, for the valuable guidance and support.

\section{Funding}

SK is thankful to (NU/DR/NUFR2/NUCSER/2019-20/04) for financially funding this research.

\section{Data availability}

The data that support the findings of this study are available from the corresponding author upon reasonable request.

\section{References}

1. Sun D, Nassal M (2006) Stable HepG2- and Huh7-based human hepatoma cell lines for efficient regulated expression of infectious hepatitis B virus. J Hepatol 45:636-645.

https://doi.org/10.1016/j.jhep.2006.05.019 
2. Jang J-W, Song Y, Kim KM et al (2016) Hepatocellular carcinoma-targeted drug discovery through image-based phenotypic screening in co-cultures of HCC cells with hepatocytes. BMC Cancer 16:810. https://doi.org/10.1186/s12885-016-2816-x

3. Habib A, Sathish V, Mallik S, Khoda B (2018) 3D printability of alginate-carboxymethyl cellulose hydrogel. Materials (Basel) 11:E454. https://doi.org/10.3390/ma11030454

4. Kapałczyńska M, Kolenda T, Przybyła W et al (2018) 2D and 3D cell cultures - a comparison of different types of cancer cell cultures. Arch Med Sci 14:910-919. https://doi.org/10.5114/aoms.2016.63743

5. Bustin SA, Benes V, Nolan T, Pfaffl MW (2005) Quantitative real-time RT-PCR - A perspective. J Mol Endocrinol 34:597-601

6. Wu X, Huang A, Xu M et al (2013) Variation of Expression Levels of Seven Housekeeping Genes at Different Life-History Stages in Porphyra yezoensis. PLoS One 8:1-8. https://doi.org/10.1371/journal.pone.0060740

7. Panina Y, Germond A, Masui S, Watanabe TM (2018) Validation of Common Housekeeping Genes as Reference for qPCR Gene Expression Analysis during iPS Reprogramming Process. Sci Rep 8:1-8. https://doi.org/10.1038/s41598-018-26707-8

8. Matsuzaki Y, Umemoto T, Tanaka Y et al (2015) $\beta 2$-Microglobulin is an appropriate reference gene for RT-PCR-based gene expression analysis of hematopoietic stem cells. Regen Ther 1:91-97. https://doi.org/10.1016/j.reth.2015.04.003

9. Yan W, Xie M, Li R et al (2020) Identification and validation of reference genes selection in ovarian cancer exposed to hypoxia. Onco Targets Ther 13:7423-7431. https://doi.org/10.2147/OTT.S249733

10. Su X, Yao X, Sun Z et al (2016) Optimization of Reference Genes for Normalization of qRT-PCR Results in Senescence Study of Mesenchymal Stem Cells. Stem Cells Dev 25:1-45. https://doi.org/10.1089/scd.2016.0031

11. Sikand K, Singh J, Ebron JS, Shukla GC (2012) Housekeeping Gene Selection Advisory: Glyceraldehyde-3-Phosphate Dehydrogenase (GAPDH) and $\beta$-Actin Are Targets of miR-644a. PLoS One 7:1-9. https://doi.org/10.1371/journal.pone.0047510

12. Chaicharoenaudomrung N, Kunhorm P, Noisa P (2019) Three-dimensional cell culture systems as an in vitro platform for cancer and stem cell modeling. World J Stem Cells 11:1065-1083

13. Foldager CB, Munir S, Ulrik-vinther M et al (2009) Validation of suitable house keeping genes for hypoxia-cultured human chondrocytes. BMC Mol Biol 10:1-8. https://doi.org/10.1186/1471-219910-94

14. Chaicharoenaudomrung N, Kunhorm P, Promjantuek W et al (2019) Fabrication of 3D calciumalginate scaffolds for human glioblastoma modeling and anticancer drug response evaluation. $J$ Cell Physiol 234:20085-20097. https://doi.org/10.1002/jcp.28608

15. Poojari R, Kini S, Srivastava R, Panda D (2015) A Chimeric Cetuximab-Functionalized Corona as a Potent Delivery System for Microtubule-Destabilizing Nanocomplexes to Hepatocellular Carcinoma 
Cells: A Focus on EGFR and Tubulin Intracellular Dynamics. Mol Pharm 12:3908-3923. https://doi.org/10.1021/acs.molpharmaceut.5b00337

16. Eckerdt F, Alvarez A, Bell J et al (2016) A simple, low-cost staining method for rapid-throughput analysis of tumor spheroids. Biotechniques 60:43-46. https://doi.org/10.2144/000114372

17. Badekila AK, Kini S, Jaiswal AK (2020) Fabrication techniques of biomimetic scaffolds in threedimensional cell culture: A review. J Cell Physiol 236:jcp.29935. https://doi.org/10.1002/jcp.29935

18. Lauretti F, Lucas De Melo F, Benati FJ et al (2003) Use of acridine orange staining for the detection of rotavirus RNA in polyacrylamide gels. J Virol Methods 114:29-35.

https://doi.org/10.1016/j.jviromet.2003.08.005

19. Rio DC, Ares M, Hannon GJ, Nilsen TW (2010) Purification of RNA using TRIzol (TRI Reagent). Cold Spring Harb Protoc 5:pdb.prot5439. https://doi.org/10.1101/pdb.prot5439

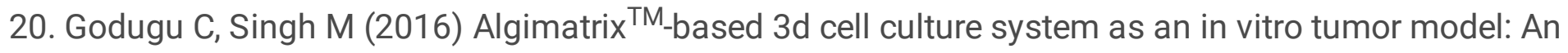
important tool in cancer research. In: Methods in Molecular Biology. Humana Press Inc., pp 117-128

21. Andersen CL, Jensen JL, Ørntoft TF (2004) Normalization of real-time quantitative reverse transcription-PCR data: A model-based variance estimation approach to identify genes suited for normalization, applied to bladder and colon cancer data sets. Cancer Res 64:5245-5250.

https://doi.org/10.1158/0008-5472.CAN-04-0496

22. Vandesompele J, De Preter K, Pattyn F et al (2002) Accurate normalization of real-time quantitative RT-PCR data by geometric averaging of multiple internal control genes. Genome Biol 3:research0034.1. https://doi.org/10.1186/gb-2002-3-7-research0034

23. Hussain H, SR L, Ahmad S et al (2019) Determination of cell viability using acridine orange/propidium iodide dual-spectrofluorometry assay. Cogent Food Agric 5:1582398. https://doi.org/10.1080/23311932.2019.1582398

24. Barui A, Khare R, Dhara S et al (2014) Ex vivo bio-compatibility of honey-alginate fibrous matrix for $\mathrm{HaCaT}$ and 3T3 with prime molecular expressions. J Mater Sci Mater Med 25:2659-2667. https://doi.org/10.1007/s10856-011-4456-7

25. Andersen T, Auk-Emblem P, Dornish M (2015) 3D Cell Culture in Alginate Hydrogels. Microarrays 4:133-161. https://doi.org/10.3390/microarrays4020133

26. Sreedharan SP, Kumar A, Giridhar P (2018) Primer design and amplification efficiencies are crucial for reliability of quantitative PCR studies of caffeine biosynthetic N-methyltransferases in coffee. 3 Biotech 8:467. https://doi.org/10.1007/s13205-018-1487-5

27. Valente V, Teixeira SA, Neder L et al (2009) Selection of suitable housekeeping genes for expression analysis in glioblastoma using quantitative RT-PCR. BMC Mol Biol 10:17. https://doi.org/10.1186/1471-2199-10-17

28. Zhang JY, Zhang F, Hong CQ et al (2015) Critical protein GAPDH and its regulatory mechanisms in cancer cells. Cancer Biol Med 12:10-22 
29. Tzanakakis ES, Hansen LK, Hu WS (2001) The role of actin filaments and microtubules in hepatocyte spheroid self-assembly. Cell Motil Cytoskeleton 48:175-189. https://doi.org/10.1002/10970169(200103)48:3<175::AID-CM1007>3.0.C0;2-2

30. Gubern C, Hurtado O, Rodríguez R et al (2009) Validation of housekeeping genes for quantitative realtime PCR in in-vivo and in-vitro models of cerebral ischaemia. BMC Mol Biol 10:57. https://doi.org/10.1186/1471-2199-10-57

31. Bian Z, Yu Y, Quan C et al (2015) RPL13A as a reference gene for normalizing mRNA transcription of ovarian cancer cells with paclitaxel and 10-hydroxycamptothecin treatments. Mol Med Rep 11:31883194. https://doi.org/10.3892/mmr.2014.3108

32. Robledo D, Hernández-Urcera J, Cal RM et al (2014) Analysis of qPCR reference gene stability determination methods and a practical approach for efficiency calculation on a turbot (Scophthalmus maximus) gonad dataset. BMC Genom 15:648. https://doi.org/10.1186/1471-216415-648

33. Krishnan SundaramIDV, Kumar Sampathkumar N, Massaad C, Grenier J (2019) Optimal use of statistical methods to validate reference gene stability in longitudinal studies. PLoS One 16:e0219440. https://doi.org/10.1371/journal.pone.0219440

\section{Figures}




\section{Acridine Orange Staining}
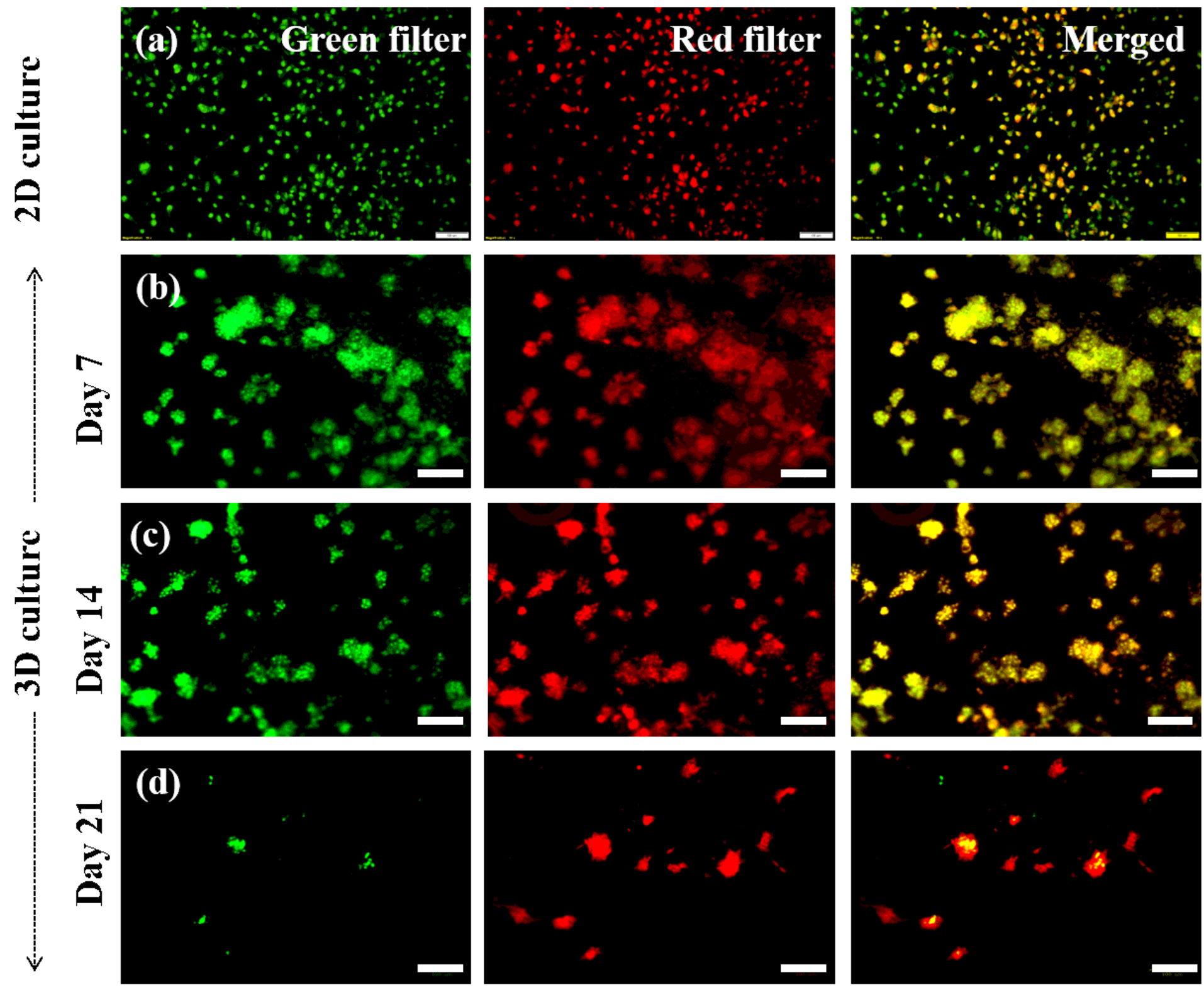

Figure 1

Fluorescent microscopic images of acridine orange-stained hepatocellular carcinoma cells cultured in 2D and alginate-carboxymethylcellulose based 3D culture; (a) 2D culture, (b) spheroids on day 7, (c) day 14, and (d) day 21 (10X magnification, and $150 \mu \mathrm{m}$ scale) 3D cultured cells. 

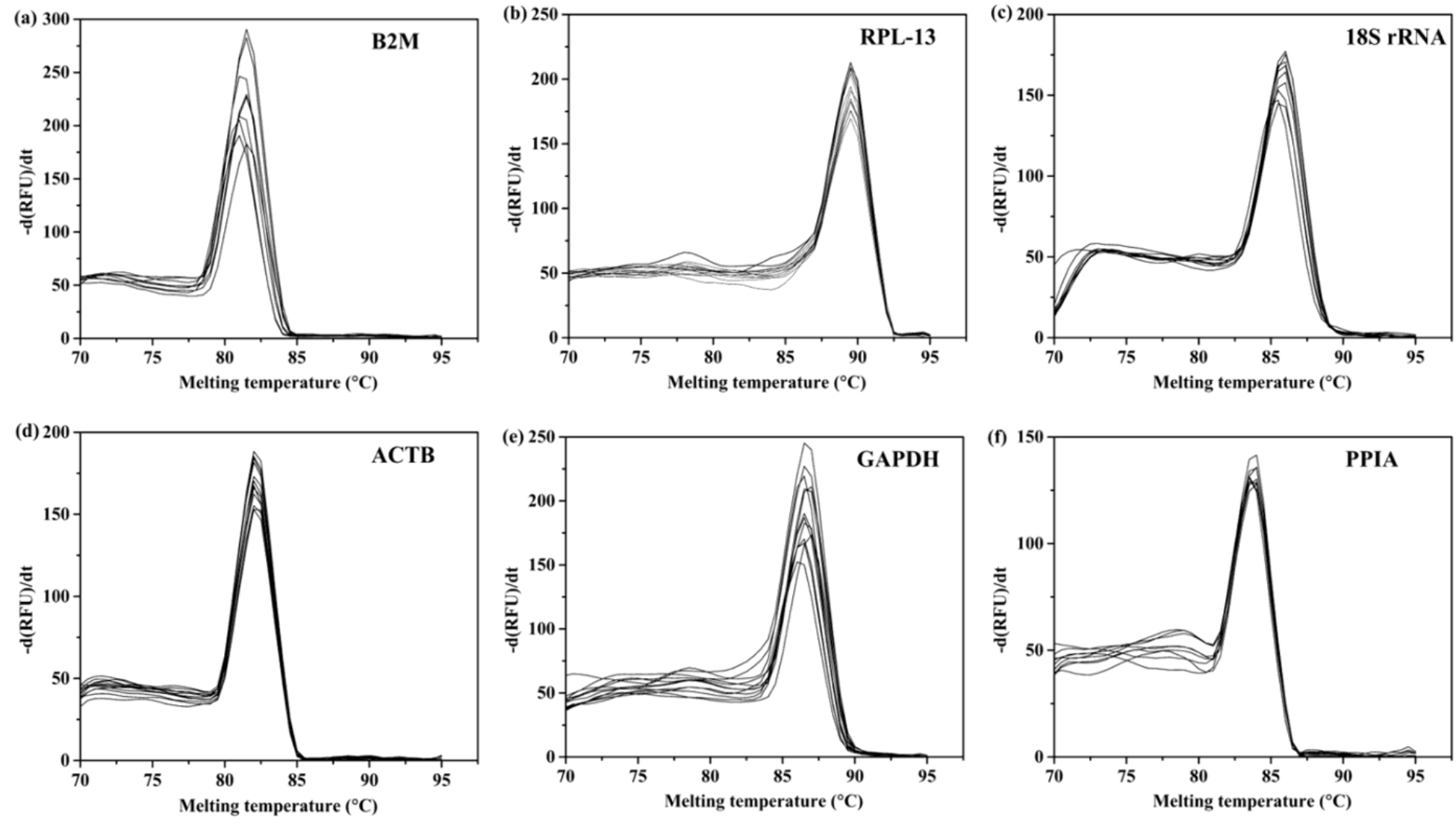

Figure 2

Melting curve analysis from a RT-qPCR assay of candidate $\neg$ housekeeping genes of hepatocellular carcinoma cell line in three-dimensional cell culture. The melting curves for the genes, (a) B2M, (b) RPL13, (c) 18S rRNA, (d) ACTB, (e) GAPDH, and (f) PPIA shows a single fluorescence peak at a constant melting temperature indicating the specificity of respective primers used in RT-qPCR.
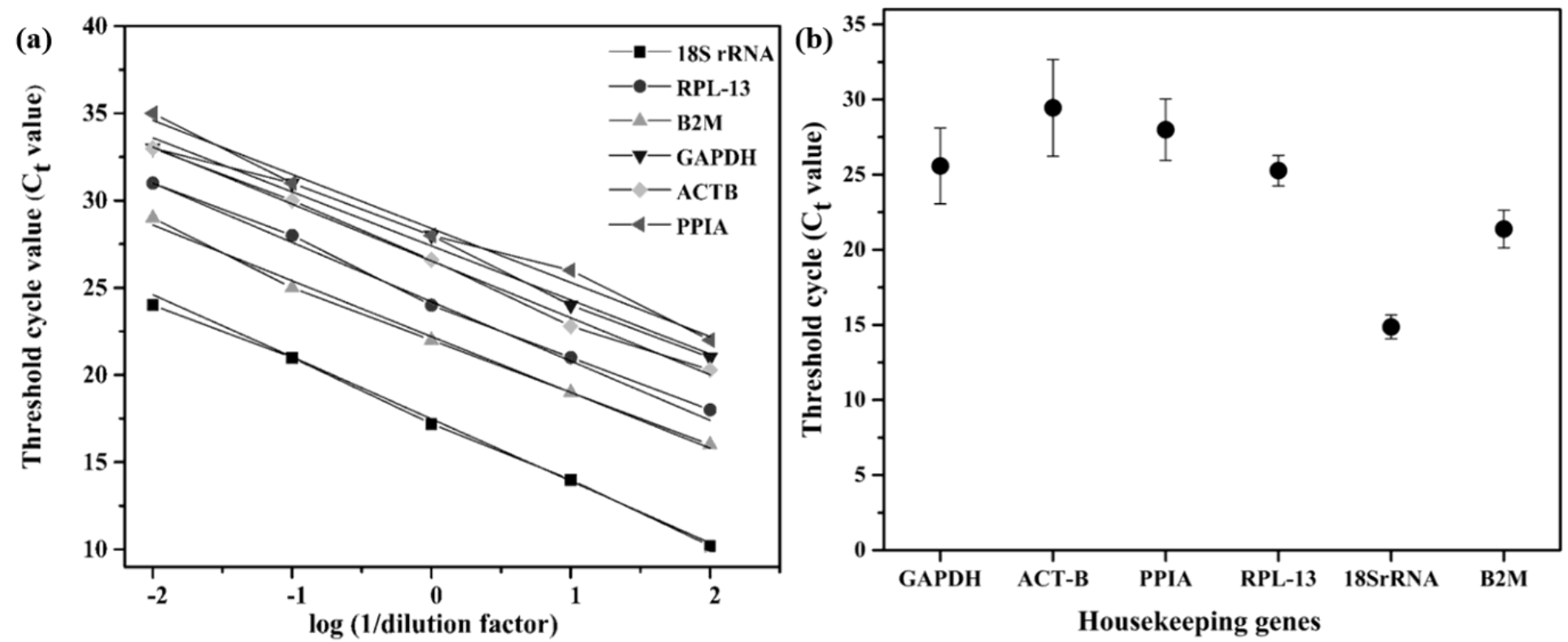

Figure 3 
Primer efficiency and mean threshold cycle values of the housekeeping genes. (a) shows the amplification efficiency of primers represented as cycle threshold values $(\mathrm{Ct})$ versus log of primer concentrations. (b) An interval plot of the mean threshold cycle values (Mean (95\% of class interval (Cl): lower-upper value)) of the housekeeping genes (GAPDH, ACT-B, PPIA, RPL-13, B2M and 18S rRNA). Values of the three replicates for four sample types (2D, 3D-7, 14, and 21 days old culture) are given. The cycle threshold values for RPL-13 were identified to be consistent for a span of 21 days in a 3D culture.
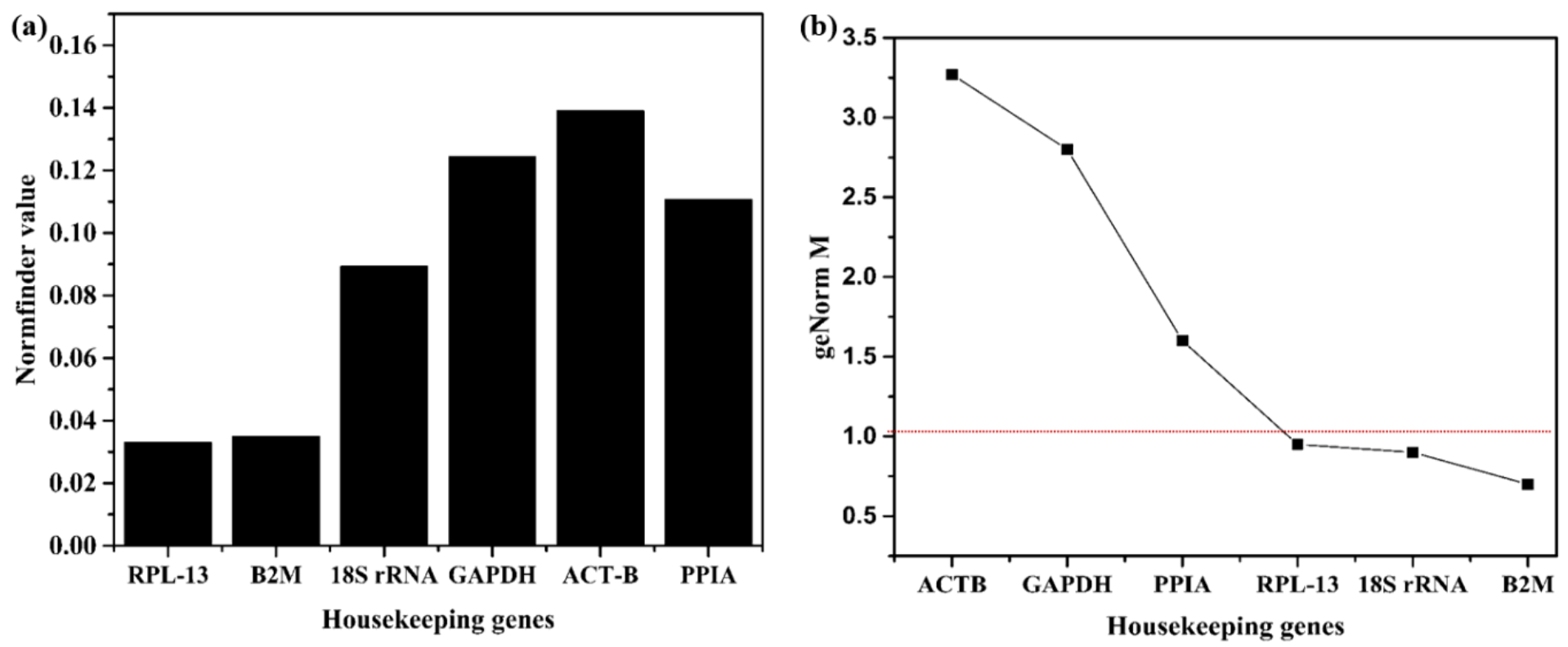

Figure 4

NormFinder and geNorm analysis of the housekeeping genes based on their stability. (a) NormFinder and (b) geNorm analysis of candidate housekeeping genes in variable culture conditions.

\section{Supplementary Files}

This is a list of supplementary files associated with this preprint. Click to download.

- supplementaryInformation.docx 